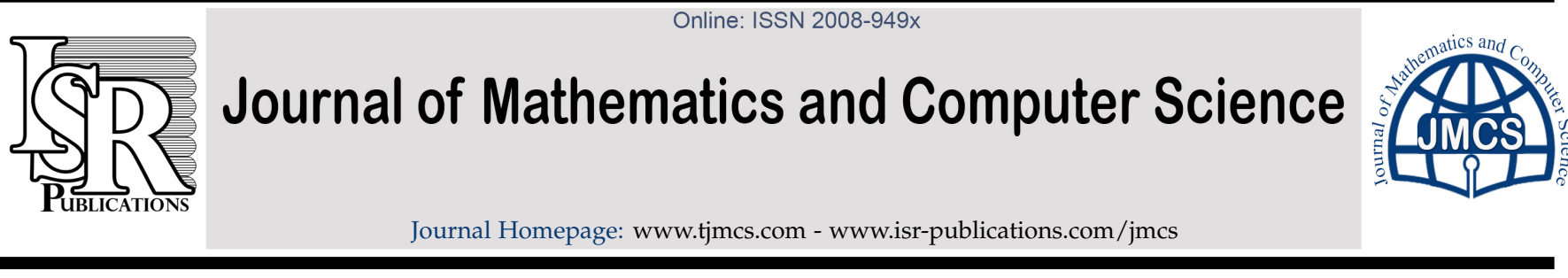

\title{
Parameters identification and dual synchronization between different chaotic and hyperchaotic systems
}

\author{
A. Othman Almatroud ${ }^{a, *}$, M. S. M. Noorani ${ }^{b}$, M. Mossa Al-sawalha ${ }^{a}$ \\ a Mathematics Department, Faculty of Science, University of Hail, Kingdom of Saudi Arabia. \\ ${ }^{b}$ School of Mathematical Sciences, Universiti Kebangsaan Malaysia, 43600 UKM Bangi, Selangor, Malaysia.
}

\begin{abstract}
This paper investigates the adaptive dual synchronization of completely different four chaotic and hyperchaotic systems with unknown parameters. Based on the Lyapunov stability theory, an efficient adaptive synchronization controller is constructed that converges the synchronization error signals to the origin with sufficient transient speed. Suitable adaptive laws of unknown parameters are designed that converged the estimated values of the unknown parameters to the true values of the systems parameters. Two numerical examples are presented and simulation results are derived to illustrate the effectiveness of the proposed dual synchronization approach.
\end{abstract}

Keywords: Chaos, dual synchronization, adaptive control, unknown parameters, Lyapunov stability theory.

2010 MSC: 93Dxx, 93Cxx.

(C)2018 All rights reserved.

\section{Introduction}

The idea of chaos synchronization was first introduced by Pecorra and Carroll [35] in 1991. Since then, the synchronization of chaotic systems has been widely investigated in various scientific disciplines for it's theoretical as well as experimental challenges [7, 25]. Different synchronization control techniques and strategies have been developed. These include, active control [44], linear feedback control [40], nonlinear control, adaptive control, sliding mode control, projective synchronization and the lag synchronization [1-6, 15-18, 20, 27, 42, 43, 45], etc.. In practical situations, some or all parameters of a chaotic systems cannot be accessible in priori, and the effect of these uncertainties may completely break the synchronization control. The adaptive control strategy is used when some or all parameters of the chaotic systems are unknown. The significant features of the adaptive control strategy include the fast response, robustness against the uncertainties, good transient performance, and easy implementation in practical applications. Recently, an interesting synchronization phenomena discovered is the dual synchronization. In the dual synchronization, more than one pair of a drive (master) and response (slave)

\footnotetext{
*Corresponding author

Email address: othman_almatroud@yahoo.com (A. Othman Almatroud)

doi: $10.22436 /$ jmcs.018.04.02
}

Received: 2017-11-28 Revised: 2018-06-17 Accepted: 2018-09-19 
chaotic systems are synchronized using a generated signal through a linear combination of the drive systems states. An intensive research has been devoted to study the dual synchronization of chaotic systems. Tsimring and Sushchik [38] proposed the idea of dual chaos synchronization both for iterated maps and ordinary differential equations. The dual synchronization between two different pairs of chaotic oscillators using a scalar signal is studied [22], where the dual synchronization is achieved by means of specific classes of piecewise-linear maps with conditional linear coupling. In [39], the authors achieved the dual synchronization between pairs of lasers by changing the optical frequency of each laser and controlled the condition of injection locking instead of matching of internal parameters. Ning et al. [26] investigated the dual synchronization among two different chaotic systems. The authors [14] established the problem of dual synchronization using time-varying gain proportional output feedback. Gosh and Chaudhary [13] further enhanced the dual synchronization of time-delay chaotic systems. Based on the KrasovskiiLyapunov functional approach, the authors [12] proposed a nonlinear observer-based projective-dual synchronization in modulated coupled time-delayed systems. The dual synchronization is further improved for fractional-order chaotic systems [41]. Using an adaptive and nonlinear feedback control approaches [28-34], the dual synchronization and anti-synchronization are investigated. To the best of our knowledge, the dual synchronization for pairs of non-identical unknown chaotic and hyperchaotic systems has not been investigated and has been remained an open problem. In addition, it is well-known that if the systems are completely different, then the messages of secure applications are more effective and can not be captured easily. Generally, the applications of chaos dual synchronization in secure communication make it much more important. In fact, in engineering, it is hardly the case that every component can be assumed to be identical. Being motivated by the above discussion, the main objective of this paper is to study the dual synchronization between two different unknown chaotic and hyperchaotic systems. Based on the Lyapunov stability theory, a suitable adaptive controller is constructed that accomplished the asymptotic stability of the synchronization errors at the origin. This paper also designed the update laws for the estimation of unknown parameters. Two numerical examples are presented to show the efficiency and performance of the proposed dual synchronization strategy. The rest of the paper is organized as follows. Section 2 presents the problem statement and theory of the proposed dual synchronization strategy. In Section 3, the dual synchronization among four unknown chaotic Lorenz, Lü, Chen and Genesio systems and dual synchronization among four unknown hyperchaotic Lorenz, Chen, Lü and Lorenz-Stenflo systems are investigated. This paper concludes in Section 5.

\section{Problem statement}

Consider the following two different systems with uncertain parameters as the drive systems:

$$
\dot{x}_{1}=f_{1}\left(x_{1}\right)+F_{1}\left(x_{1}\right) \alpha, \quad \dot{y}_{1}=g_{1}\left(y_{1}\right)+G_{1}\left(y_{1}\right) \beta,
$$

where $x_{1} \in R^{n}, y_{1} \in R^{n}$ are the state vectors of the systems, $f_{1}: R^{n} \rightarrow R^{n}$ and $g_{1}: R^{n} \rightarrow R^{n}$ are two continuous vector functions, $F_{1}: R^{n} \rightarrow R^{n \times m_{1}}, G_{1}: R^{n} \rightarrow R^{n \times q_{1}}$ are two matrix functions, and $\alpha \in R^{m_{1}}$, $\beta \in R^{q_{1}}$ are the unknown parameter vectors of the two drive systems. By a linear combination of the drive systems states, a scalar signal is generated in the form of

$$
\varepsilon_{d}=\sum_{i=1}^{n}\left(a_{i} x_{1}+b_{i} y_{1}\right)=A^{\top} x_{1}+B^{\top} y_{1}=C^{\top} x,
$$

where $A=\left(a_{1}, a_{2}, \ldots, a_{n}\right)^{\top}$ and $B=\left(b_{1}, b_{2}, \ldots, b_{n}\right)^{\top}$ are coupling parameters to be selected later, $C=$ $\left(A^{\top} B^{\top}\right)^{\top}, x=\left(x_{1}^{\top} y_{1}^{\top}\right)^{\top}$. This generated scalar signal is fed to the following response systems which are completely different from the drive systems.

$$
\dot{x}_{2}=f_{2}\left(x_{2}\right)+F_{2}\left(x_{2}\right) \delta+u_{1}, \quad \dot{y}_{2}=g_{2}\left(y_{2}\right)+G_{2}\left(y_{2}\right) \theta+u_{2},
$$


where $x_{2} \in R^{n}$ and $y_{2} \in R^{n}$ are the state vectors, $f_{2}: R^{n} \rightarrow R^{n}$ and $g_{2}: R^{n} \rightarrow R^{n}$ are two continuous vector functions, $F_{2}: R^{n} \rightarrow R^{n \times m_{2}}, G_{2}: R^{n} \rightarrow R^{n \times q_{2}}$ are two matrix functions, and $\delta \in R^{m_{2}}, \theta \in R^{q_{2}}$ are the unknown parameter vectors of the two response systems and $u=\left(\begin{array}{lll}u_{1} & u_{2}\end{array}\right)^{\top} \in R^{2 n}$ is a controller. The feedback signal of the system (2.2) is given as follows:

$$
\varepsilon_{r}=\sum_{i=1}^{n}\left(a_{i} x_{2}+b_{i} y_{2}\right)=C^{\top} y,
$$

where $y=\left(x_{2}^{\top} y_{2}^{\top}\right)^{\top}$ is a combination of the responses states.

\subsection{Adaptive dual synchronization}

System (2.1) can be written in the following form

$$
\left[\begin{array}{l}
\dot{x}_{1} \\
\dot{y}_{1}
\end{array}\right]=\left[\begin{array}{l}
f_{1}\left(x_{1}\right) \\
g_{1}\left(y_{1}\right)
\end{array}\right]+\left[\begin{array}{cc}
F_{1}\left(x_{1}\right) & 0 \\
0 & G_{1}\left(y_{1}\right)
\end{array}\right]\left[\begin{array}{l}
\alpha \\
\beta
\end{array}\right], \quad \dot{x}=f(x)+F(x) \wedge .
$$

where $\dot{x}=\left[\begin{array}{ll}\dot{x}_{1} & \dot{y}_{1}\end{array}\right]^{\top}, f(x)=\left[\begin{array}{ll}f_{1}\left(x_{1}\right) & g_{1}\left(y_{1}\right)\end{array}\right]^{\top}, F(x)=\left[\begin{array}{cc}F_{1}\left(x_{1}\right) & 0 \\ 0 & G_{1}\left(y_{1}\right)\end{array}\right]$, and $\Lambda=\left[\begin{array}{ll}\alpha & \beta\end{array}\right]^{\top}$. Similarly, system (2.2) can be written in the following form:

$$
\left[\begin{array}{l}
\dot{x}_{2} \\
\dot{y}_{2}
\end{array}\right]=\left[\begin{array}{l}
f_{2}\left(x_{2}\right) \\
g_{2}\left(y_{2}\right)
\end{array}\right]+\left[\begin{array}{cc}
F_{2}\left(x_{2}\right) & 0 \\
0 & G_{2}\left(y_{2}\right)
\end{array}\right]\left[\begin{array}{l}
\delta \\
\theta
\end{array}\right]+\left[\begin{array}{l}
u_{1} \\
u_{2}
\end{array}\right], \quad \dot{y}=g(y)+G(y) x+u,
$$

where $\dot{y}=\left[\begin{array}{ll}\dot{x}_{2} & \dot{y}_{2}\end{array}\right]^{\top}, g(y)=\left[\begin{array}{ll}f_{2}\left(x_{2}\right) & g_{2}\left(y_{2}\right)\end{array}\right]^{\top}, G(x)=\left[\begin{array}{cc}F_{2}\left(x_{1}\right) & 0 \\ 0 & G_{2}\left(y_{2}\right)\end{array}\right]$, and $x=\left[\begin{array}{ll}\delta & \theta\end{array}\right]^{\top}$ and $u=\left[\begin{array}{ll}u_{1} & u_{2}\end{array}\right]^{\top}$. The main goal is to dual synchronize the drive systems (2.1) and the respond systems (2.2) such that

$$
\lim _{t \rightarrow \infty}\left\|x_{2}-x_{1}\right\|=0, \quad \lim _{t \rightarrow \infty}\left\|y_{2}-y_{1}\right\|=0 .
$$

Remark 2.1. In this paper, we will assume that systems (2.1) and systems (2.2) satisfies $f_{1}(\cdot) \neq f_{2}(\cdot)$ and $\mathrm{F}_{1}(\cdot) \neq \mathrm{F}_{2}(\cdot), \mathrm{g}_{1}(\cdot) \neq \mathrm{g}_{2}(\cdot)$ and $\mathrm{G}_{1}(\cdot) \neq \mathrm{G}_{2}(\cdot)$, then the structure of systems (2.1) and (2.2) are different.

Theorem 2.2. If the adaptive control functions is selected as

$$
u=f(x)+F(x) \hat{\Lambda}-g(y)-G(y) \hat{x}-k e-e_{s}
$$

and updated by the following adaptation laws:

$$
\dot{\hat{\Lambda}}=-[\mathrm{F}(\mathrm{x})]^{\top} e, \quad \dot{\hat{\chi}}=[\mathrm{G}(\mathrm{y})]^{\top} e,
$$

then the response system (2.3) can adaptive dual synchronize the drive system (2.4) globally and asymptotically, where $\mathrm{k}>0$ is a constant, $\mathrm{e}_{\mathrm{s}}=\mathrm{C}^{\mathrm{T}}(\mathrm{y}-\mathrm{x})$ is linear coupling for dual synchronization, and $\hat{\Lambda}, \hat{\mathrm{x}}$ are the estimations of the unknown parameters $\Lambda, x$, which need to be estimated.

Proof. The error system of the adaptive control scheme between systems (2.3) and (2.4) is given as follows:

$$
\dot{e}=G(y)(x-\hat{\chi})-F(x)(\Lambda-\hat{\Lambda})-e k-e_{s} .
$$

Let us construct the following Lyapunov function candidate as

$$
V=\frac{1}{2}\left[e^{\top} e+\tilde{\Lambda}^{\top} \tilde{\Lambda}+\tilde{\chi}^{\top} \tilde{\chi}\right]
$$

where, $\tilde{\Lambda}=\Lambda-\hat{\Lambda}, \tilde{\chi}=\chi-\hat{\chi}$. Taking the time derivative of $V$ along the error dynamical system (2.5)which 
is given as follows:

$$
\begin{aligned}
\dot{V} & =\left[e^{\top} \dot{e}+\dot{\tilde{\Lambda}}^{\top} \tilde{\Lambda}+\dot{\tilde{\chi}}^{\top} \tilde{\chi}\right], \\
& =e^{\top}\left[G(y) \tilde{\chi}-F(x) \tilde{\Lambda}-k e-e_{s}\right]+e^{T} F(x) \tilde{\Lambda}-e^{\top} G(x) \tilde{\chi}=-e^{\top} e k-e^{\top} e_{s}=-e^{T} P e .
\end{aligned}
$$

Obviously, to ensure that the zero solution of the error dynamical system (2.5) is asymptotically stable, the real symmetric matrix P should be positive definite. So, based on Lyapunov stability theory [36], we know that the error dynamical system (2.5) is asymptotically stable.

\section{Dual synchronization of different chaotic systems with unknown parameters}

In this section of the paper, we imply our proposed adaptive control method to achieve the asymptotical dual synchronization of non identical chaotic systems with unknown parameters. Let us consider the drive-response systems as follows:

Drive 1: Lorenz system [23] is given by

$$
\dot{x_{1}}=\sigma\left(y_{1}-x_{1}\right), \quad \dot{y_{1}}=\rho x_{1}-x_{1} z_{1}-y_{1}, \quad \dot{z_{1}}=x_{1} y_{1}-\gamma z_{1} .
$$

Drive 2: Lü system [24] is given by

$$
\dot{x_{2}}=\alpha\left(y_{2}-x_{2}\right), \quad \dot{y_{2}}=-x_{2} z_{2}+\delta y_{2}, \quad \dot{z_{2}}=x_{2} y_{2}-\beta z_{2} .
$$

Response 1: Chen system [9]

$$
\dot{x_{3}}=\alpha_{1}\left(y_{3}-x_{3}\right)+u_{1}, \quad \dot{y_{3}}=\left(\delta_{1}-\alpha_{1}\right) x_{3}-x_{3} z_{3}+\delta_{1} y_{3}+u_{2}, \quad \dot{z_{3}}=x_{3} y_{3}-\beta_{1} z_{3}+u_{3} .
$$

Response 2: Gensio system [11]

$$
\dot{x_{4}}=y_{4}+u_{4}, \quad \dot{y_{4}}=z_{4}+u_{5}, \quad \dot{z_{4}}=-\sigma_{1} x_{4}-\rho_{1} y_{4}-\gamma_{1} z_{4}+x_{4}^{2}+u_{6},
$$

where $\sigma, \rho, \gamma, \alpha, \delta, \beta$ and $\alpha_{1}, \delta_{1}, \beta_{1}, \sigma_{1}, \rho_{1}, \gamma_{1}$ are unknown system parameters which need to be estimated. The control input is defined as $u_{i},(i=1,2, \ldots, 6)$. Subtracting (3.1) from (3.3) and (3.2) from (3.4), then the error system is given as follows:

$$
\begin{aligned}
& \dot{e_{1}}=\alpha_{1}\left(y_{3}-x_{3}\right)-\sigma\left(y_{1}-x_{1}\right)+u_{1}, \\
& \dot{e_{2}}=\left(\delta_{1}-\alpha_{1}\right) x_{3}-x_{3} z_{3}+\delta_{1} y_{3}-\rho x_{1}+x_{1} z_{1}+y_{1}+u_{2}, \\
& \dot{e_{3}}=x_{3} y_{3}-\beta_{1} z_{3}-x_{1} y_{1}+\gamma z_{1}+u_{3}, \\
& \dot{e_{4}}=y_{4}-\alpha\left(y_{2}-x_{2}\right)+u_{4}, \\
& \dot{e_{5}}=z_{4}+x_{2} z_{2}-\delta y_{2}+u_{5}, \\
& \dot{e_{6}}=-\sigma_{1} x_{4}-\rho_{1} y_{4}-\gamma_{1} z_{4}+x_{4}^{2}-x_{2} y_{2}+\beta z_{2}+u_{6},
\end{aligned}
$$

where $e_{1}=x_{3}-x_{1}, e_{2}=y_{3}-y_{1}, e_{3}=z_{3}-z_{1}, e_{4}=x_{4}-x_{2}, e_{5}=y_{4}-y_{2}, e_{6}=z_{4}-z_{2}$. The dual synchronization of non identical chaotic systems needs the stabilization of the error system (3.5) at the origin by a suitable adaptive controller which forces the trajectories of the response systems to track the trajectories of the drive systems so that the error vector in the situation of unknown parameter i.e., $\lim _{t \rightarrow \infty}\left\|e_{i}(t)\right\|=0,(i=1,2, \ldots, 6)$. In order to achieve that, we state the following theorem.

Theorem 3.1. For any initial conditions, the systems (3.1)-(3.2) and (3.3)-(3.4) are globally asymptotically dual synchronized if we design the following adaptive control functions and adaptation law:

$$
\begin{aligned}
& u_{1}=-\hat{\alpha}_{1}\left(y_{3}-x_{3}\right)+\hat{\sigma}\left(y_{1}-x_{1}\right)-k_{1} e_{1}-e_{s}, \\
& u_{2}=-\left(\hat{\delta}_{1}-\hat{\alpha}_{1}\right) x_{3}+x_{3} z_{3}-\hat{\delta}_{1} y_{3}+\hat{\rho} x_{1}-x_{1} z_{1}-y_{1}-k_{2} e_{2}-e_{s}, \\
& u_{3}=-x_{3} y_{3}+\hat{\beta}_{1} z_{3}+x_{1} y_{1}-\hat{\gamma} z_{1}-k_{3} e_{3}-e_{s} \\
& u_{4}=-y_{4}+\hat{\alpha}\left(y_{2}-x_{2}\right)-k_{4} e_{4}-e_{s} \\
& u_{5}=-z_{4}-x_{2} z_{2}+\hat{\delta} y_{2}-k_{5} e_{5}-e_{s}, \\
& u_{6}=\hat{\sigma}_{1} x_{4}+\hat{\rho}_{1} y_{4}+\hat{\gamma}_{1} z_{4}-x_{4}^{2}+x_{2} y_{2}-\hat{\beta} z_{2}-k_{6} e_{6}-e_{s},
\end{aligned}
$$


and

$$
\begin{array}{clll}
\dot{\hat{\sigma}}=-\left(y_{1}-x_{1}\right) e_{1}, & \dot{\hat{\rho}}=-x_{1} e_{2}, & \dot{\hat{\gamma}}=z_{1} e_{3}, & \dot{\hat{\alpha}}=-\left(y_{2}-x_{2}\right) e_{4}, \\
\dot{\hat{\delta}}=y_{2} e_{5}, & \dot{\hat{\beta}}=z_{2} e_{6}, & \dot{\hat{\alpha}}_{1}=\left(y_{3}-x_{3}\right) e_{1}-x_{3} e_{2}, & \dot{\hat{\delta}}_{1}=\left(x_{3}+y_{3}\right) e_{2}, \\
\hat{\beta}_{1}=-z_{3} e_{3}, & \dot{\hat{\sigma}}_{1}=-x_{4} e_{6}, & \dot{\hat{\rho}}_{1}=-y_{4} e_{6}, & \dot{\hat{\gamma}}_{1}=-z_{4} e_{6},
\end{array}
$$

where $k_{i}>0,(i=1,2, \ldots, 6), \hat{\sigma}, \hat{\rho}, \hat{\gamma}, \hat{\alpha}, \hat{\delta}, \hat{\beta}$ and $\hat{\alpha}_{1}, \hat{\delta}_{1}, \hat{\beta}_{1}, \hat{\sigma}_{1}, \hat{\rho}_{1}, \hat{\gamma}_{1}$ are the parameter estimations of $\sigma, \rho, \gamma$, $\alpha, \delta, \beta$ and $\alpha_{1}, \delta_{1}, \beta_{1}, \sigma_{1}, \rho_{1}, \gamma_{1}$, respectively, and

$$
e_{s}=\sum_{i=1}^{3} a_{i} e_{i}+\sum_{i=1}^{3} b_{i} e_{j}, \quad j=4,5,6, e=y-x, y \neq x .
$$

where, $e_{\mathrm{s}}$ is the linear coupling of the drive and response systems.

Proof. Inserting (3.6) into (3.5) yields the following form of the error system

$$
\begin{aligned}
& \dot{e_{1}}=\tilde{\alpha}_{1}\left(y_{3}-x_{3}\right)-\tilde{\sigma}\left(y_{1}-x_{1}\right)-k_{1} e_{1}-e_{s}, \\
& \dot{e_{2}}=\left(\tilde{\delta}_{1}-\tilde{\alpha}_{1}\right) x_{3}+\tilde{\delta}_{1} y_{3}-\rho x_{1}-k_{2} e_{2}-e_{s}, \\
& \dot{e_{3}}=-\tilde{\beta}_{1} z_{3}+\tilde{\gamma} z_{1}-k_{3} e_{3}-e_{s}, \\
& \dot{e_{4}}=-\tilde{\alpha}\left(y_{2}-x_{2}\right)-k_{4} e_{4}-e_{s}, \\
& \dot{e_{5}}=-\tilde{\delta} y_{2}-k_{5} e_{5}-e_{s}, \\
& \dot{e_{6}}=-\tilde{\sigma}_{1} x_{4}-\tilde{\rho}_{1} y_{4}-\tilde{\gamma}_{1} z_{4}+\tilde{\beta} z_{2}-k_{6} e_{6}-e_{s},
\end{aligned}
$$

where $\tilde{\sigma}=\sigma-\hat{\sigma}, \tilde{\rho}=\rho-\hat{\rho}, \tilde{\gamma}=\gamma-\hat{\gamma}, \tilde{\alpha}=\alpha-\hat{\alpha}, \tilde{\delta}=\delta-\hat{\delta}, \tilde{\beta}=\beta-\hat{\beta}$ and $\tilde{\alpha}_{1}=\alpha_{1}-\hat{\alpha}_{1}, \tilde{\delta}_{1}=\delta_{1}-\hat{\delta}_{1}, \tilde{\beta}_{1}=$ $\beta_{1}-\hat{\beta}_{1}, \tilde{\sigma}_{1}=\sigma_{1}-\hat{\sigma}_{1}, \tilde{\rho}_{1}=\rho_{1}-=\hat{\rho}_{1}, \tilde{\gamma}_{1}=\gamma_{1}-\hat{\gamma}_{1}$. Let us construct the following Lyapunov function candidate as

$$
V=\frac{1}{2}\left(e^{\top} e+\tilde{\sigma}^{2}+\tilde{\rho}^{2}+\tilde{\gamma}^{2}+\tilde{\alpha}^{2}+\tilde{\delta}^{2}+\tilde{\beta}^{2}+\tilde{\alpha}_{1}^{2}+\tilde{\delta}_{1}^{2}+\tilde{\beta}_{1}^{2}+\tilde{\sigma}_{1}^{2}+\tilde{\rho}_{1}^{2}+\tilde{\gamma}_{1}^{2}\right)
$$

Applying the above equations in the time derivative of $\mathrm{V}$ leads to

$$
\begin{aligned}
\dot{V}= & e^{\top} \dot{e}+\tilde{\sigma} \dot{\tilde{\sigma}}+\tilde{\rho} \dot{\tilde{\rho}}+\tilde{\gamma} \dot{\tilde{\gamma}}+\tilde{\alpha} \dot{\tilde{\alpha}}+\tilde{\delta} \tilde{\tilde{\delta}}+\tilde{\beta} \dot{\tilde{\beta}}+\tilde{\alpha}_{1} \dot{\tilde{\alpha}}_{1}+\tilde{\delta}_{1} \dot{\tilde{\delta}}_{1}+\tilde{\beta}_{1} \dot{\tilde{\beta}}_{1}+\tilde{\sigma}_{1} \dot{\tilde{\sigma}}_{1}+\tilde{\rho}_{1} \dot{\tilde{\rho}}_{1}+\tilde{\gamma}_{1} \dot{\tilde{\gamma}}_{1} \\
= & e_{1}\left[\tilde{\alpha}_{1}\left(y_{3}-x_{3}\right)-\tilde{\sigma}\left(y_{1}-x_{1}\right)-k_{1} e_{1}-e_{s}\right]+e_{2}\left[\left(\tilde{\delta}_{1}-\tilde{\alpha}_{1}\right) x_{3}+\tilde{\delta}_{1} y_{3}\right. \\
& \left.-\tilde{\rho} x_{1}-k_{2} e_{2}-e_{s}\right]+e_{3}\left[-\tilde{\beta}_{1} z_{3}+\tilde{\gamma} z_{1}-k_{3} e_{3}-e_{s}\right]+e_{4}\left[-\tilde{\alpha}\left(y_{2}-x_{2}\right)\right. \\
& \left.-k_{4} e_{4}-e_{s}\right]+e_{5}\left[-\tilde{\delta} y_{2}-k_{5} e_{5}-e_{s}\right]+e_{6}\left[-\tilde{\sigma}_{1} x_{4}-\tilde{\rho}_{1} y_{4}-\tilde{\gamma}_{1} z_{4}+\tilde{\beta} z_{2}\right. \\
& \left.-k_{6} e_{6}-e_{s}\right]+\tilde{\sigma}\left(\left(y_{1}-x_{1}\right) e_{1}\right)+\tilde{\rho}\left(x_{1} e_{2}\right)+\tilde{\gamma}\left(-z_{1} e_{3}\right)+\tilde{\alpha}\left(\left(y_{2}-x_{2}\right) e_{4}\right) \\
& +\tilde{\delta}\left(-y_{2} e_{5}\right)+\tilde{\beta}\left(-z_{2} e_{6}\right)+\tilde{\alpha}_{1}\left(-\left(\left(y_{3}-x_{3}\right) e_{1}-x_{3} e_{2}\right)\right)+\tilde{\delta}_{1}\left(-\left(x_{3}+y_{3}\right) e_{2}\right) \\
& +\tilde{\beta}_{1}\left(z_{3} e_{3}\right)+\tilde{\sigma}_{1}\left(x_{4} e_{6}\right)+\tilde{\rho}_{1}\left(y_{4} e_{6}\right)+\tilde{\gamma}_{1}\left(z_{4} e_{6}\right) \\
= & -\left[\left(k_{1}+a_{1}\right) e_{1}^{2}+\left(a_{1}+a_{2}\right) e_{1} e_{2}+\left(a_{1}+a_{3}\right) e_{1} e_{3}+\left(a_{1}+b_{1}\right) e_{1} e_{4}+\left(a_{1}+b_{2}\right) e_{1} e_{5}\right. \\
& +\left(a_{1}+b_{3}\right) e_{1} e_{6}+\left(k_{2}+a_{2}\right) e_{2}^{2}+\left(a_{2}+a_{3}\right) e_{2} e_{3}+\left(a_{2}+b_{1}\right) e_{2} e_{4}+\left(a_{2}+b_{2}\right) e_{2} e_{5} \\
& +\left(a_{2}+b_{3}\right) e_{2} e_{6}+\left(k_{3}+a_{3}\right) e_{3}^{2}+\left(a_{3}+b_{1}\right) e_{3} e_{4}+\left(a_{3}+b_{2}\right) e_{3} e_{5}+\left(a_{3}+b_{3}\right) e_{3} e_{6} \\
& \left.+\left(k_{4}+b_{1}\right) e_{4}^{2}+\left(b_{1}+b_{2}\right) e_{4} e_{5}+\left(b_{1}+b_{3}\right) e_{4} e_{6}+\left(k_{5}+b_{2}\right) e_{5}^{2}+\left(b_{2}+b_{3}\right) e_{5} e_{6}+\left(k_{6}+b_{3}\right) e_{6}^{2}\right] \\
= & -e^{\top} P e_{1}
\end{aligned}
$$

where, $e=\left[\left|e_{1}\right|,\left|e_{2}\right|,\left|e_{3}\right|,\left|e_{4}\right|,\left|e_{5}\right|,\left|e_{6}\right|\right]$ and $P$ is real symmetric. From the Lyapunov theorem of stability [19], it is simple to point out that the zero equilibrium point $\left(e_{i}=0, i=1, \ldots, 6\right)$ of the error dynamical system (3.5) is globally asymptotic stable if the real symmetric matrix $\mathrm{P}$ is positive definite. According 
to Sylvester's theorem [36], $\mathrm{P}$ is positive definite if and only if $\Delta_{i}>0, i=1,2, \ldots, 6$, where $\Delta_{i}$ represents the $i$ th order sequential sub determinant of matrix. That is, we should choose the appropriate coupled parameters. Then, we realize the adaptive dual synchronization between different chaotic systems. This completes the proof.

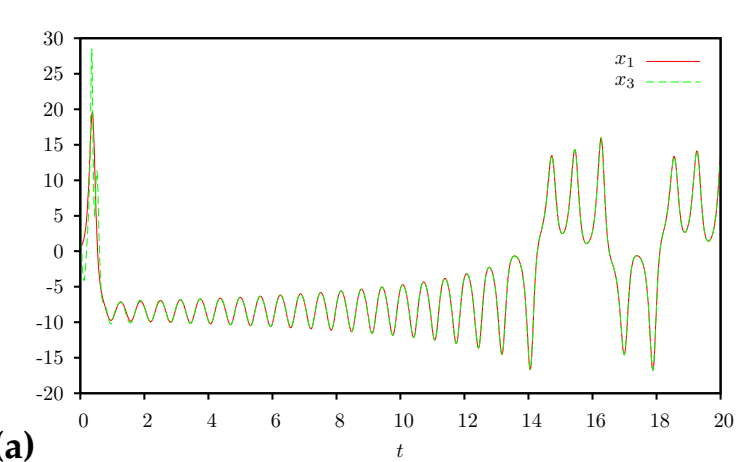

(a)
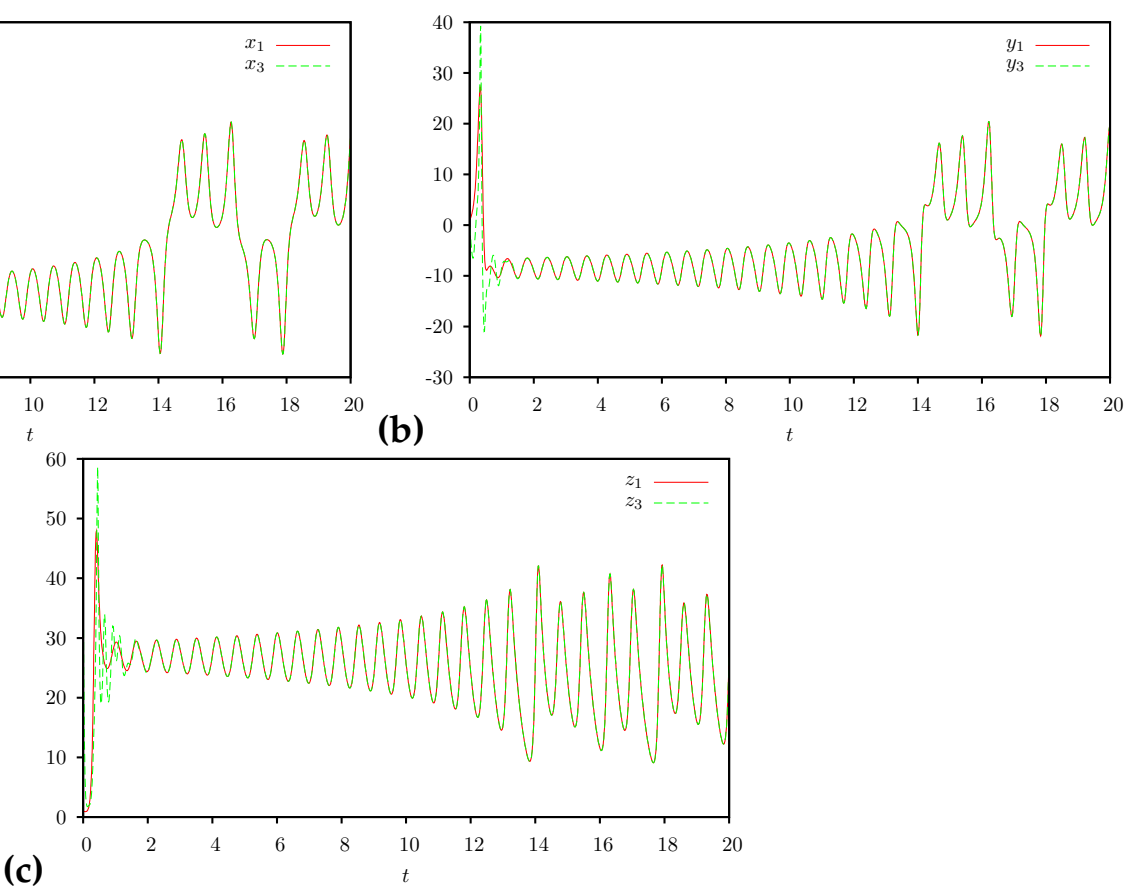

Figure 1: Evolution of the drive system (3.1) and response system (3.3) state, (a) signals $x_{1}$ and $x_{3} ;$ (b) signals $y_{1}$ and $y_{3} ;$ (c) signals $z_{1}$ and $z_{3}$.

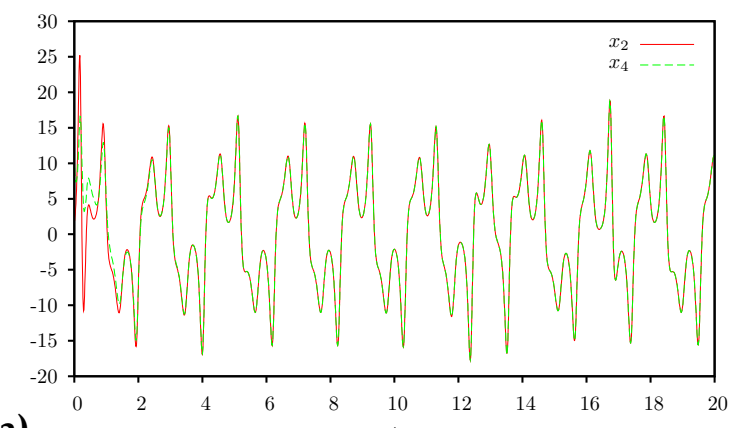

(a)
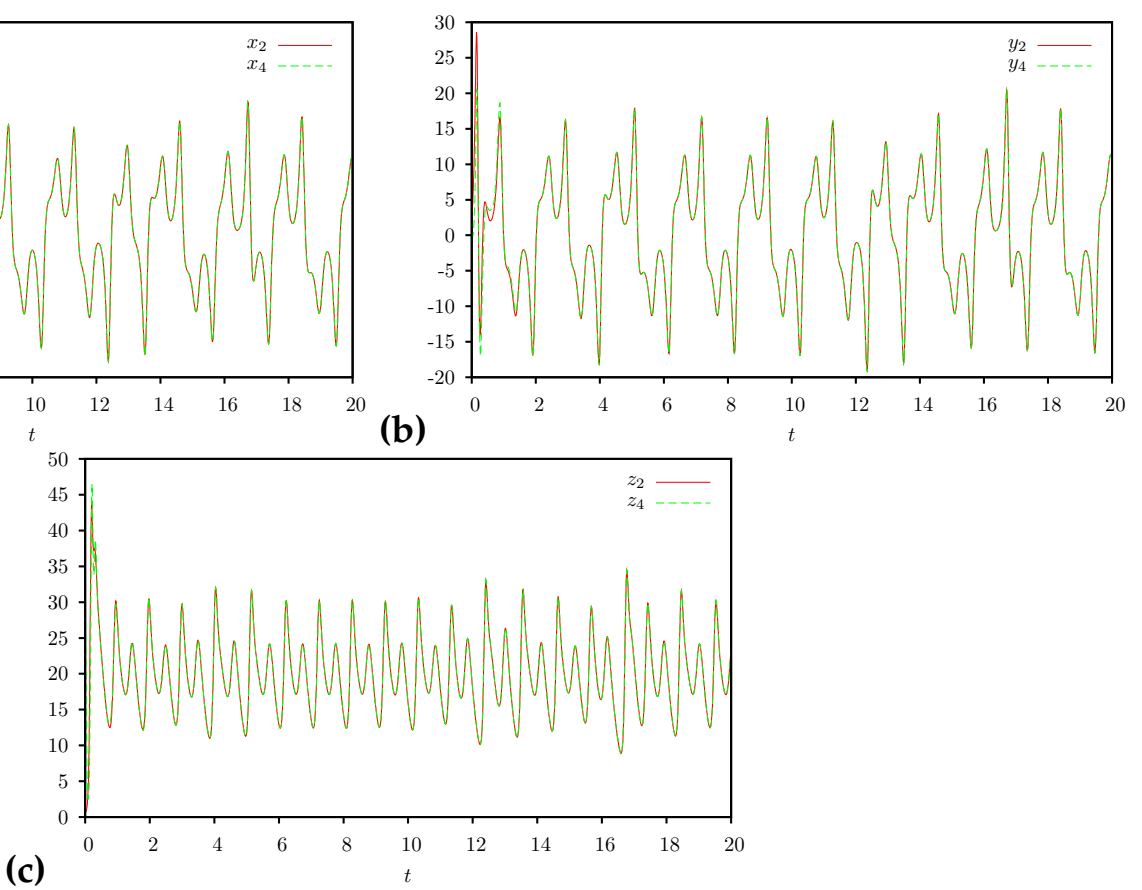

Figure 2: Evolution of the drive system (3.2) and response (3.4) system state, (a) signals $x_{2}$ and $x_{4}$; (b) signals $y_{2}$ and $y_{4} ;(\mathbf{c})$ signals $z_{2}$ and $z_{4}$. 
(a)

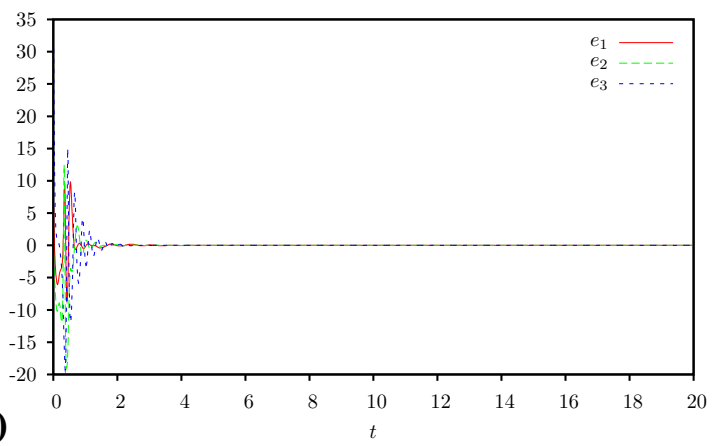

(b)

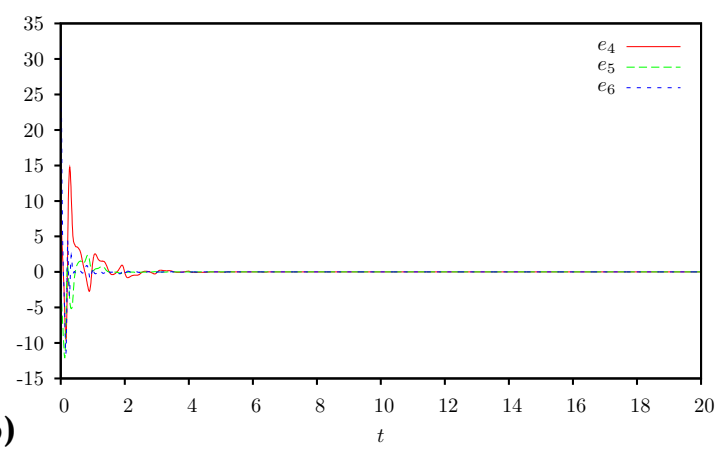

Figure 3: The dual synchronization errors (a) $e_{1}, e_{2}, e_{3}$ between the pair of systems (3.1) and (3.3); (b) $e_{4}, e_{5}, e_{6}$ between the pair of systems (3.2) and (3.4).

(a)
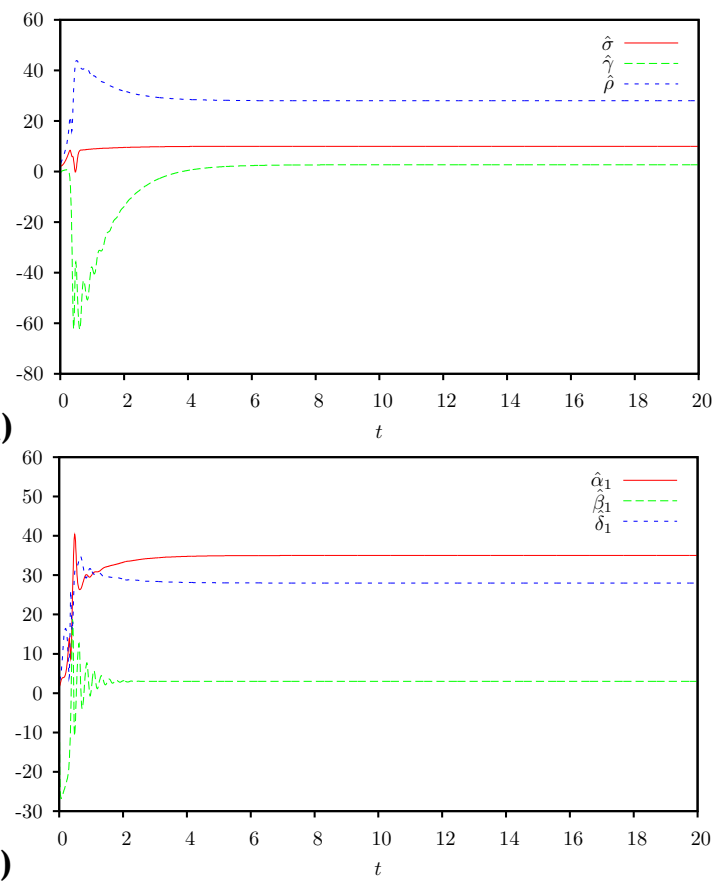

(b)
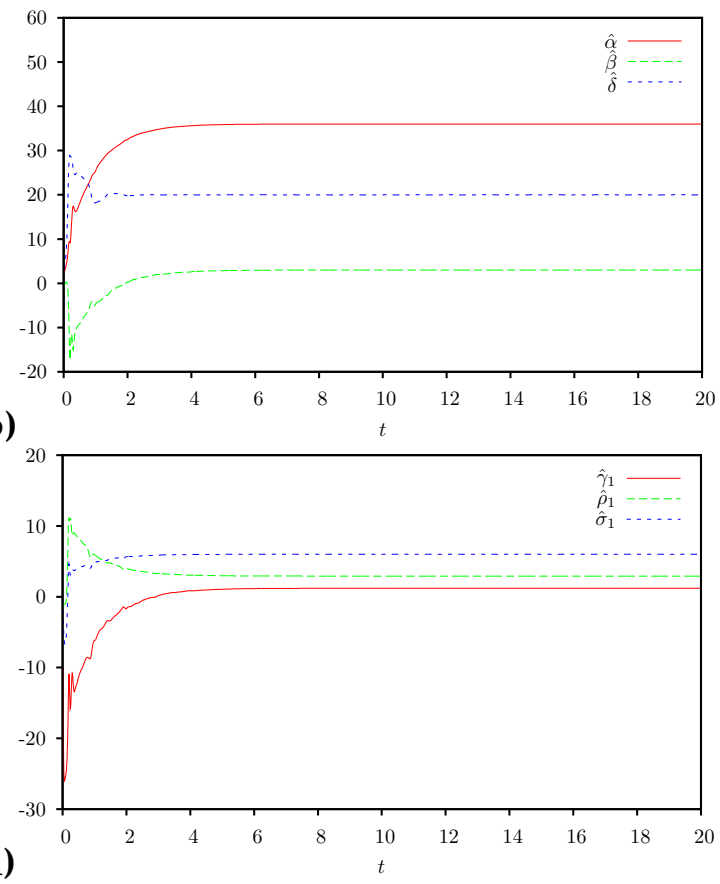

Figure 4: (a) Estimate values of parameters of system (3.1); (b) Estimate values of parameters of system (3.2); (c) Estimate values of parameters of system (3.3); (d) Estimate values of parameters of system (3.4).

\subsection{Numerical simulations}

In this subsection, to verify and demonstrate the effectiveness of the proposed method, we discuss the simulation results for the adaptive dual synchronization of different chaotic systems. In the numerical simulations, the fourth-order Runge-Kutta method is used to solve the systems (3.1)-(3.4) with time step size 0.001 . The parameters of the drive and the response systems are set as $\sigma=10, \rho=28, \gamma=8 / 3, \alpha=$ $36, \delta=20, \beta=3, \alpha_{1}=35, \delta_{1}=28, \beta_{1}=3, \sigma_{1}=6, \rho_{1}=2.92, \gamma_{1}=1.2, a_{i}=(1,1,1), b_{i}=(1,1,1), i=1,2,3$ and $k_{i}=4, i=0,1, \ldots, 6$, for which condition $P$ is positive definite. The initial conditions of the drive and response systems are taken as, $x_{1}(0)=0.5, y_{1}(0)=1, z_{1}(0)=1$ and $x_{2}(0)=1.5, y_{2}(0)=2.5, z_{2}(0)=0.65$, $x_{3}(0)=10.5, y_{3}(0)=1, z_{3}(0)=37, x_{4}(0)=10.5, y_{4}(0)=1, z_{4}(0)=37$, respectively. Adaptive dual synchronization of the systems (3.1)-(3.4) via adaptive control law (3.6) and (3.7) with the initial estimated parameters $\hat{\sigma}(0)=2, \hat{\rho}(0)=3, \hat{\gamma}(0)=-1, \hat{\alpha}(0)=2, \hat{\delta}(0)=3, \hat{\beta}(0)=-1$ and $\hat{\alpha}_{1}(0)=2, \hat{\delta}_{1}(0)=3, \hat{\beta}_{1}(0)=$ $-1, \hat{\sigma}_{1}(0)=2, \hat{\rho}_{1}(0)=3, \hat{\gamma}_{1}(0)=-1$ are shown in Figures 1-4. Figures 1-2 show the evolution of the drive systems (3.1)-(3.2) and the response systems (3.3)-(3.4). Figure 3 shows the error signals $e_{1}, e_{2}, e_{3}$ 
and $e_{4}, e_{5}, e_{6}$ with initial conditions $e_{1}(0)=10, e_{2}(0)=0, e_{3}(0)=36, e_{4}(0)=9, e_{5}(0)=-1.5, e_{6}(0)=36.35$. Figure 4 shows that the estimates $\hat{\sigma}, \hat{\rho}, \hat{\gamma}, \hat{\alpha}, \hat{\delta}, \hat{\beta}$ and $\hat{\alpha}_{1}, \hat{\delta}_{1}, \hat{\beta}_{1}, \hat{\sigma}_{1}, \hat{\rho}_{1}, \hat{\gamma}_{1}$ of the unknown parameters $\sigma, \rho, \gamma$, $\alpha, \delta, \beta$ and $\alpha_{1}, \delta_{1}, \beta_{1}, \sigma_{1}, \rho_{1}, \gamma_{1}$ converges to $\sigma=10, \rho=28, \gamma=8 / 3, \alpha=36, \delta=20, \beta=3$ and $\alpha_{1}=35, \delta_{1}=$ $28, \beta_{1}=3, \sigma_{1}=6, \rho_{1}=2.92, \gamma_{1}=1.2$ as $\mathrm{t} \rightarrow \infty$.

\section{Dual synchronization of different hyperchaotic systems with unknown parameters}

In this section, we will choose the four systems with different structure that are hyperchaotic systems, namely, hyperchaotic Lorenz system, hyperchaotic Chen system as the drives and hyperchaotic Lü, hyperchaotic Lorenz-Stenflo as the responses, we illustrate the effectiveness of the adaptive dual synchronization scheme of different hyperchaotic systems. The nonlinear differential equations that describe the different hyperchaotic systems are

Drive 1: Hyperchaotic Lorenz system [10] is given by

$$
\dot{x_{1}}=\alpha_{1}\left(y_{1}-x_{1}\right), \quad \dot{y_{1}}=\beta_{1} x_{1}-x_{1} z_{1}+y_{1}-w_{1}, \quad \dot{z}_{1}=x_{1} y_{1}-\gamma_{1} z_{1}, \quad \dot{w}_{1}=\theta_{1} y_{1} z_{1} .
$$

Drive 2: Hyperchaotic Chen [21] is given by

$$
\dot{x_{2}}=\alpha_{2}\left(y_{2}-x_{2}\right)+w_{2}, \quad \dot{y_{2}}=\beta_{2} x_{2}-x_{2} z_{2}+\delta_{2} y_{2}, \quad \dot{z_{2}}=x_{2} y_{2}-\gamma_{2} z_{2}, \quad \dot{w_{2}}=y_{2} z_{2}+\theta_{2} w_{2} .
$$

Response 1: Hyperchaotic Lü [8] is given by

$$
\begin{aligned}
\dot{x_{3}} & =\alpha_{3}\left(\mathrm{y}_{3}-x_{3}\right)+w_{3}+u_{1}, \\
\dot{y_{3}} & =-x_{3} z_{3}+\delta_{3} y_{3}+u_{2}, \\
\dot{z_{3}} & =x_{3} y_{3}-\gamma_{3} z_{3}+u_{3} \\
\dot{w_{3}} & =x_{3} z_{3}+\theta_{3} w_{3}+u_{4} .
\end{aligned}
$$

Response 2: Hyperchaotic Lorenz-Stenflo [37] is given by

$$
\begin{aligned}
\dot{x_{4}} & =\alpha_{4}\left(y_{4}-x_{4}\right)+\delta_{4} w_{4}+u_{5}, \\
\dot{y}_{4} & =\beta_{4} x_{4}-x_{4} z_{4}-y_{4}+u_{6}, \\
\dot{z_{4}} & =x_{4} y_{4}-\gamma_{4} z_{4}+u_{7}, \\
\dot{w_{4}} & =-x_{4}-\alpha_{4} w_{4}+u_{8},
\end{aligned}
$$

where $\alpha_{1}, \beta_{1}, \gamma_{1}, \theta_{1}, \alpha_{2}, \beta_{2}, \delta_{2}, \gamma_{2}, \theta_{2}, \alpha_{3}, \delta_{3}, \gamma_{3}, \theta_{3}, \alpha_{4}, \beta_{4}, \gamma_{4}, \delta_{4}$ are unknown system parameters. The control input is defined as $u_{i},(i=1,2, \ldots, 8)$. The error system can be written as follows:

$$
\begin{aligned}
& \dot{e_{1}}=\alpha_{3}\left(y_{3}-x_{3}\right)+w_{3}-\alpha_{1}\left(y_{1}-x_{1}\right)+u_{1}, \\
& \dot{e_{2}}=-x_{3} z_{3}+\delta_{3} y_{3}-\beta_{1} x_{1}+x_{1} z_{1}-y_{1}+w_{1}+u_{2}, \\
& \dot{e_{3}}=x_{3} y_{3}-\gamma_{3} z_{3}-x_{1} y_{1}+\gamma_{1} z_{1}+u_{3}, \\
& \dot{e_{4}}=x_{3} z_{3}+\theta_{3} w_{3}-\theta_{1} y_{1} z_{1}+u_{4} \\
& \dot{e_{5}}=\alpha_{4}\left(y_{4}-x_{4}\right)+\delta_{4} w_{4}-\alpha_{2}\left(y_{2}-x_{2}\right)-w_{2}+u_{5}, \\
& \dot{e_{6}}=\beta_{4} x_{4}-x_{4} z_{4}-y_{4}-\beta_{2} x_{2}+x_{2} z_{2}-\delta_{2} y_{2}+u_{6}, \\
& \dot{e_{7}}=x_{4} y_{4}-\gamma_{4} z_{4}-x_{2} y_{2}+\gamma_{2} z_{2}+u_{7}, \\
& \dot{e_{8}}=-x_{4}-\alpha_{4} w_{4}-y_{2} z_{2}-\theta_{2} w_{2}+u_{8},
\end{aligned}
$$

where $e_{1}=x_{3}-x_{1}, e_{2}=y_{3}-y_{1}, e_{3}=z_{3}-z_{1}, e_{4}=w_{3}-w_{3}, e_{5}=x_{4}-x_{2}, e_{6}=y_{4}-y_{2}, e_{7}=z_{4}-z_{2}$, $e_{8}=w_{4}-w_{2}$. Our goal is to find proper control functions $u_{i}, i=1,2, \ldots, 8$ and parameter update rule, such that system (4.1)-(4.2) asymptotically dual synchronization systems (4.3)-(4.4), i.e., $\lim _{t \rightarrow \infty}\left\|e_{i}(t)\right\|=0$, $(\mathrm{i}=1,2, \ldots, 8)$. The following theorem shows that hyperchaotic systems (4.1)-(4.2) and (4.3)-(4.4) can be dual synchronized effectively by the following proposed adaptive controller. 
Theorem 4.1. Systems (4.1)-(4.2) and (4.3)-(4.4) are globally asymptotically dual synchronized if we design the following adaptive control functions and adaptation law:

$$
\begin{aligned}
& \mathrm{u}_{1}=-\hat{\alpha}_{3}\left(\mathrm{y}_{3}-x_{3}\right)-w_{3}+\hat{\alpha}_{1}\left(\mathrm{y}_{1}-x_{1}\right)-\mathrm{k}_{1} e_{1}-e_{s}, \\
& \mathrm{u}_{2}=x_{3} z_{3}-\hat{\delta}_{3} y_{3}+\hat{\beta}_{1} x_{1}-x_{1} z_{1}+y_{1}-w_{1}-k_{2} e_{2}-e_{s}, \\
& u_{3}=-x_{3} y_{3}+\hat{\gamma}_{3} z_{3}+x_{1} y_{1}-\hat{\gamma}_{1} z_{1}-k_{3} e_{3}-e_{s}, \\
& u_{4}=-x_{3} z_{3}-\hat{\theta}_{3} w_{3}+\hat{\theta}_{1} y_{1} z_{1}-k_{4} e_{4}-e_{s}, \\
& u_{5}=-\hat{\alpha}_{4}\left(y_{4}-x_{4}\right)-\hat{\delta}_{4} w_{4}+\hat{\alpha}_{2}\left(y_{2}-x_{2}\right)+w_{2}-k_{5} e_{5}-e_{s}, \\
& u_{6}=-\hat{\beta}_{4} x_{4}+x_{4} z_{4}+y_{4}+\hat{\beta}_{2} x_{2}-x_{2} z_{2}+\hat{\delta}_{2} y_{2}-k_{6} e_{6}-e_{s}, \\
& u_{7}=-x_{4} y_{4}+\hat{\gamma}_{4} z_{4}+x_{2} y_{2}-\hat{\gamma}_{2} z_{2}-k_{7} e_{7}-e_{s}, \\
& u_{8}=x_{4}+\hat{\alpha}_{4} w_{4}+y_{2} z_{2}+\hat{\theta}_{2} w_{2}-k_{8} e_{8}-e_{s},
\end{aligned}
$$

and

$$
\begin{aligned}
& \dot{\hat{\alpha}}_{1}=-\left(\mathrm{y}_{1}-\mathrm{x}_{1}\right) \mathrm{e}_{1}, \quad \dot{\hat{\beta}}_{1}=-\mathrm{x}_{1} \mathrm{e}_{2}, \quad \dot{\hat{\gamma}}_{1}=z_{1} \mathrm{e}_{3}, \quad \dot{\hat{\theta}}_{1}=-\mathrm{y}_{1} z_{1} \mathrm{e}_{4}, \\
& \dot{\hat{\alpha}}_{2}=-\left(\mathrm{y}_{2}-\mathrm{x}_{2}\right) \mathrm{e}_{5}, \quad \dot{\hat{\beta}}_{2}=-\mathrm{x}_{2} \mathrm{e}_{6}, \quad \dot{\hat{\delta}}_{2}=-\mathrm{y}_{2} \mathrm{e}_{6}, \quad \dot{\hat{\gamma}}_{2}=\mathrm{x}_{2} \mathrm{e}_{7}, \\
& \dot{\hat{\theta}}_{2}=-w_{2} e_{8} \quad \dot{\hat{\alpha}}_{3}=\left(y_{3}-x_{3}\right) e_{1}, \quad \dot{\hat{\delta}}_{3}=y_{3} e_{2}, \quad \dot{\hat{\gamma}}_{3}=-z_{3} e_{3}, \\
& \dot{\hat{\theta}}_{3}=w_{3} e_{4}, \quad \dot{\hat{\alpha}}_{4}=\left(y_{4}-x_{4}\right) e_{5}-w_{4} e_{8}, \quad \dot{\hat{\beta}}_{4}=x_{4} e_{6}, \quad \dot{\hat{\gamma}}_{4}=-z_{4} e_{7}, \quad \dot{\hat{\delta}}_{4}=w_{2} e_{5},
\end{aligned}
$$

where $k_{i}>0,(i=1,2, \ldots, 8), \hat{\alpha}_{1}, \hat{\beta}_{1}, \hat{\gamma}_{1}, \hat{\theta}_{1}, \hat{\alpha}_{2}, \hat{\beta}_{2}, \hat{\delta}_{2}, \hat{\gamma}_{2}, \hat{\theta}_{2}, \hat{\alpha}_{3}, \hat{\delta}_{3}, \hat{\gamma}_{3}, \hat{\theta}_{3}, \hat{\alpha}_{4}, \hat{\beta}_{4}, \hat{\gamma}_{4}, \hat{\delta}_{4}$ are the parameter estimations of $\alpha_{1}, \beta_{1}, \gamma_{1}, \theta_{1}, \alpha_{2}, \beta_{2}, \delta_{2}, \gamma_{2}, \theta_{2}, \alpha_{3}, \delta_{3}, \gamma_{3}, \theta_{3}, \alpha_{4}, \beta_{4}, \gamma_{4}, \delta_{4}$, respectively, and

$$
e_{s}=a_{1} e_{1}+a_{2} e_{2}+a_{3} e_{3}+a_{4} e_{4}+b_{1} e_{5}+b_{2} e_{6}+b_{3} e_{6}+b_{4} e_{8} .
$$

Proof. The error system (4.5) can be written as follows

$$
\begin{aligned}
& \dot{e_{1}}=\tilde{\alpha}_{3}\left(y_{3}-x_{3}\right)+w_{3}-\tilde{\alpha}_{1}\left(y_{1}-x_{1}\right)-k_{1} e_{1}-e_{s}, \\
& \dot{e_{2}}=\tilde{\delta}_{3} y_{3}-\tilde{\beta}_{1} x_{1}-k_{2} e_{2}-e_{s}, \\
& \dot{e_{3}}=-\tilde{\gamma}_{3} z_{3}+\tilde{\gamma}_{1} z_{1}-k_{3} e_{3}-e_{s}, \\
& \dot{e_{4}}=\tilde{\theta}_{3} w_{3}-\tilde{\theta}_{1} y_{1} z_{1}-k_{4} e_{4}-e_{s}, \\
& \dot{e_{5}}=\tilde{\alpha}_{4}\left(y_{4}-x_{4}\right)+\tilde{\delta}_{4} w_{4}-\tilde{\alpha}_{2}\left(y_{2}-x_{2}\right)-k_{5} e_{5}-e_{s}, \\
& \dot{e_{6}}=\tilde{\beta}_{4} x_{4}-\tilde{\beta}_{2} x_{2}-\tilde{\delta}_{2} y_{2}-k_{6} e_{6}-e_{s}, \\
& \dot{e_{7}}=-\tilde{\gamma}_{4} z_{4}+\tilde{\gamma}_{2} z_{2}-k_{7} e_{7}-e_{s}, \\
& \dot{e_{8}}=-\tilde{\alpha}_{4} w_{4}-\tilde{\theta}_{2} w_{2}-k_{8} e_{8}-e_{s},
\end{aligned}
$$

where, $\tilde{\alpha}_{1}=\alpha_{1}-\hat{\alpha}_{1}, \tilde{\beta}_{1}=\beta_{1}-\hat{\beta}_{1}, \tilde{\gamma}_{1}=\gamma_{1}-\hat{\gamma}_{1}, \tilde{\theta}_{1}=\theta_{1}-\hat{\theta}_{1}, \tilde{\alpha}_{2}=\alpha_{2}-\hat{\alpha}_{2}, \tilde{\beta}_{2}=\beta_{2}-\hat{\beta}_{2}, \tilde{\delta}_{2}=\delta_{2}-\hat{\delta}_{2}, \tilde{\gamma}_{2}=$ $\gamma_{2}-\hat{\gamma}_{2}, \tilde{\theta}_{2}=\theta_{2}-\hat{\theta}_{2}, \tilde{\alpha}_{3}=\alpha_{3}-\hat{\alpha}_{3}, \tilde{\delta}_{3}=\delta_{3}-\hat{\delta}_{3}, \tilde{\gamma}_{3}=\gamma_{3}-\hat{\gamma}_{3}, \tilde{\theta}_{3}=\theta_{3}-\hat{\theta}_{3}, \tilde{\alpha}_{4}=\alpha_{4}-\hat{\alpha}_{4}, \tilde{\beta}_{4}=\beta_{4}-\hat{\beta}_{4}, \tilde{\gamma}_{4}=$ $\gamma_{4}-\hat{\gamma}_{4}, \tilde{\delta}_{4}=\delta_{4}-\hat{\delta}_{4}$. The Lyapunov function candidate is formed as

$$
\mathrm{V}=\frac{1}{2}\left(e^{\top} e+\tilde{\alpha}_{1}^{2}+\tilde{\beta}_{1}^{2}+\tilde{\gamma}_{1}^{2}+\tilde{\theta}_{1}^{2}+\tilde{\alpha}_{2}^{2}+\tilde{\beta}_{2}^{2}+\tilde{\delta}_{2}^{2}+\tilde{\gamma}_{2}^{2}+\tilde{\theta}_{2}^{2}+\tilde{\alpha}_{3}^{2}+\tilde{\delta}_{3}^{2}+\tilde{\gamma}_{3}^{2}+\tilde{\theta}_{3}^{2}+\tilde{\alpha}_{4}^{2}+\tilde{\beta}_{4}^{2}+\tilde{\gamma}_{4}^{2}+\tilde{\delta}_{4}^{2}\right)
$$

Applying the above equations in the time derivative of $\mathrm{V}$ leads to the time derivative of $\mathrm{V}$ leads to

$$
\begin{aligned}
\dot{V}= & e^{\top} \dot{e}+\tilde{\alpha}_{1} \dot{\tilde{\alpha}}_{1}+\tilde{\beta}_{1} \dot{\tilde{\beta}}_{1}+\tilde{\gamma}_{1} \dot{\tilde{\gamma}}_{1}+\tilde{\theta}_{1} \dot{\tilde{\theta}}_{1}+\tilde{\alpha}_{2} \dot{\tilde{\alpha}}_{2}+\tilde{\beta}_{2} \dot{\tilde{\beta}}_{2}+\tilde{\delta}_{2} \dot{\tilde{\delta}}_{2}+\tilde{\gamma}_{2} \dot{\tilde{\gamma}}_{2}+\tilde{\theta}_{2} \dot{\tilde{\theta}}_{2} \\
& +\tilde{\alpha}_{3} \dot{\tilde{\alpha}}_{3}+\tilde{\delta}_{3} \dot{\tilde{\delta}}_{3}+\tilde{\gamma}_{3} \dot{\tilde{\gamma}}_{3}+\tilde{\theta}_{3} \dot{\tilde{\theta}}_{3}+\tilde{\delta}_{4} \dot{\tilde{\delta}}_{4}+\tilde{\beta}_{4} \tilde{\tilde{\beta}}_{4}+\tilde{\gamma}_{4} \dot{\tilde{\gamma}}_{4}+\tilde{\delta}_{4} \dot{\tilde{\delta}}_{4} \\
= & e_{1}\left[\tilde{\alpha}_{3}\left(\mathrm{y}_{3}-x_{3}\right)-\tilde{\alpha}_{1}\left(\mathrm{y}_{1}-x_{1}\right)-\mathrm{k}_{1} e_{1}-e_{s}\right]+e_{2}\left[\tilde{\delta}_{3} y_{3}-\tilde{\beta}_{1} x_{1}-k_{2} e_{2}\right. \\
& \left.-e_{s}\right]+e_{3}\left[-\tilde{\gamma}_{3} z_{3}+\tilde{\gamma}_{1} z_{1}-k_{3} e_{3}-e_{s}\right]+e_{4}\left[\tilde{\theta}_{3} w_{3}-\tilde{\theta}_{1} y_{1} z_{1}-k_{4} e_{4}-e_{s}\right]
\end{aligned}
$$




$$
\begin{aligned}
& +e_{5}\left[\tilde{\alpha}_{4}\left(y_{4}-x_{4}\right)+\tilde{\delta}_{4} w_{4}-\tilde{\alpha}_{2}\left(y_{2}-x_{2}\right)-k_{5} e_{5}-e_{s}\right]+e_{6}\left[\tilde{\beta}_{4} x_{4}-\tilde{\beta}_{2} x_{2}\right. \\
& \left.-\tilde{\delta}_{2} y_{2}-k_{6} e_{6}-e_{s}\right]+e_{7}\left[-\tilde{\gamma}_{4} z_{4}+\tilde{\gamma}_{2} z_{2}-k_{7} e_{7}-e_{s}\right]+e_{8}\left[-\tilde{\alpha}_{4} w_{4}-\tilde{\theta}_{2} w_{2}\right. \\
& \left.-k_{8} e_{8}-e_{s}\right]+\tilde{\alpha}_{1}\left(\left(y_{1}-x_{1}\right) e_{1}\right)+\tilde{\beta}_{1}\left(x_{1} e_{2}\right)+\tilde{\gamma}_{1}\left(-z_{1} e_{3}\right)+\tilde{\theta}_{1}\left(y_{1} z_{1} e_{4}\right) \\
& +\tilde{\alpha}_{2}\left(\left(y_{2}-x_{2}\right) e_{5}\right)+\tilde{\beta}_{2}\left(x_{2} e_{6}\right)+\tilde{\delta}_{2}\left(y_{2} e_{6}\right)+\tilde{\gamma}_{2}\left(-z_{2} e_{7}\right)+\tilde{\theta}_{2}\left(w_{2} e_{8}\right) \\
& +\tilde{\alpha}_{3}\left(-\left(y_{3}-x_{3}\right) e_{1}\right)+\tilde{\delta}_{3}\left(-y_{3} e_{2}\right)+\tilde{\gamma}_{3}\left(z_{3} e_{3}\right)+\tilde{\theta}_{3}\left(-w_{3} e_{4}\right) \\
& +\tilde{\alpha}_{4}\left(-\left(y_{4}-x_{4}\right) e_{5}-w_{4} e_{8}\right)+\tilde{\beta}_{4}\left(-x_{4} e_{6}\right)+\tilde{\gamma}_{4}\left(z_{4} e_{7}\right)+\tilde{\delta}_{4}\left(-w_{2} e_{5}\right) \\
& =-\left[\left(k_{1}+a_{1}\right) e_{1}^{2}+\left(a_{1}+a_{2}\right) e_{1} e_{2}+\left(a_{1}+a_{3}\right) e_{1} e_{3}+\left(a_{1}+a_{4}\right) e_{1} e_{4}+\left(a_{1}+b_{1}\right) e_{1} e_{5}\right. \\
& +\left(a_{1}+b_{2}\right) e_{1} e_{6}+\left(a_{1}+b_{3}\right) e_{1} e_{7}+\left(a_{1}+b_{4}\right) e_{1} e_{8}+\left(k_{2}+a_{2}\right) e_{2}^{2}+\left(a_{2}+a_{3}\right) e_{2} e_{3} \\
& +\left(a_{2}+a_{4}\right) e_{2} e_{4}+\left(a_{2}+b_{1}\right) e_{2} e_{5}+\left(a_{2}+b_{2}\right) e_{2} e_{6}+\left(a_{2}+b_{3}\right) e_{2} e_{7}+\left(a_{2}+b_{4}\right) e_{2} e_{8} \\
& +\left(k_{3}+a_{3}\right) e_{3}^{2}+\left(a_{3}+a_{4}\right) e_{3} e_{4}-\left(a_{3}+b_{1}\right) e_{3} e_{5}+\left(a_{3}+b_{2}\right) e_{3} e_{6}+\left(a_{3}+b_{3}\right) e_{3} e_{7} \\
& +\left(a_{3}+b_{3}\right) e_{3} e_{8}+\left(k_{4}+a_{4}\right) e_{4}^{2}+\left(a_{4}+b_{1}\right) e_{4} e_{5}+\left(a_{4}+b_{2}\right) e_{4} e_{6}+\left(a_{4}+b_{3}\right) e_{4} e_{7} \\
& +\left(a_{4}+b_{4}\right) e_{4} e_{8}+\left(k_{5}+b_{1}\right) e_{5}^{2}+\left(b_{1}+b_{2}\right) e_{5} e_{6}+\left(b_{1}+b_{3}\right) e_{5} e_{7}+\left(b_{1}+b_{4}\right) e_{5} e_{8} \\
& +\left(k_{6}+b_{2}\right) e_{6}^{2}+\left(b_{2}+b_{3}\right) e_{6} e_{7}+\left(b_{2}+b_{4}\right) e_{6} e_{8}+\left(k_{7}+b_{3}\right) e_{7}^{2}+\left(b_{3}+b_{4}\right) e_{7} e_{8} \\
& \left.+\left(k_{8}+b_{4}\right) e_{8}^{2}\right] \\
& =-e^{\top} P e \text {, }
\end{aligned}
$$

where, $e_{i}>0,(i=1,2, \ldots 8)$ and $P$ is is real symmetric. From the Lyapunov theorem of stability [19], it is simple to point out that the zero equilibrium point $\left(e_{i}=0, i=1, \ldots, 8\right)$ of the error dynamical system (4.5) is globally asymptotic stable if the real symmetric matrix $\mathrm{P}$ is positive definite. According to Sylvester's theorem [36], $\mathrm{P}$ is positive definite if and only if $\Delta_{i}>0, i=1,2, \ldots, 8$, where $\Delta_{i}$ represents the ith order sequential sub determinant of matrix. That is, we should choose the appropriate coupled parameters. Then, we realize the adaptive dual synchronization between different hyperchaotic systems. This completes the proof.

(a)
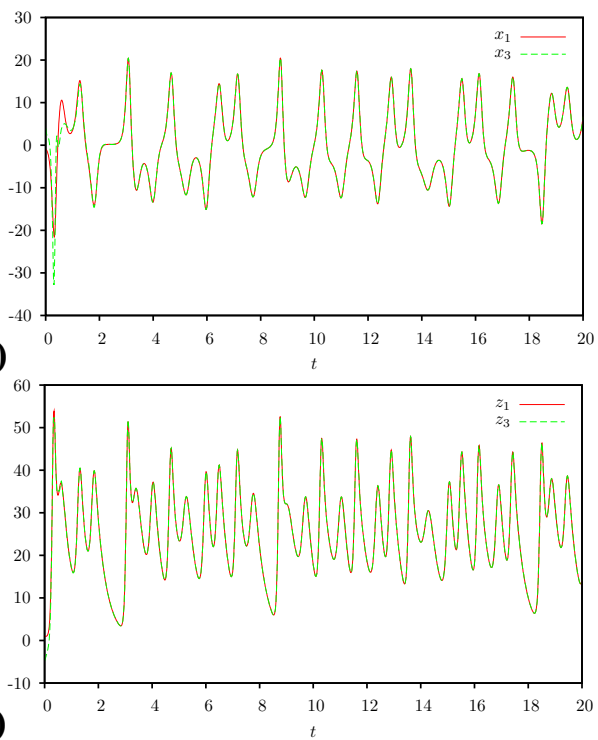

(b)
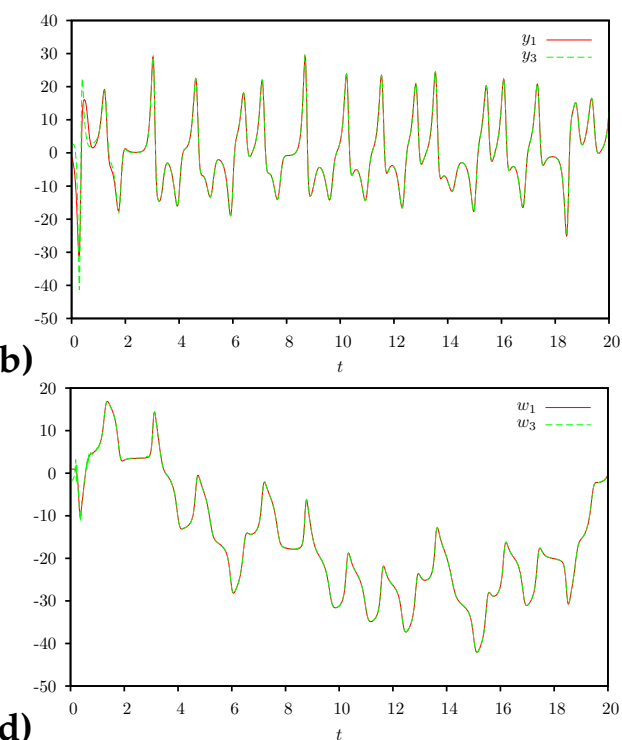

Figure 5: Evolution of the drive system (4.1) and response system (4.3) state, (a) signals $x_{1}$ and $x_{3}$; (b) signals $y_{1}$ and $y_{3} ;(\mathbf{c})$ signals $z_{1}$ and $z_{3} ;(\mathbf{d})$ signals $w_{1}$ and $w_{3}$. 
(a)
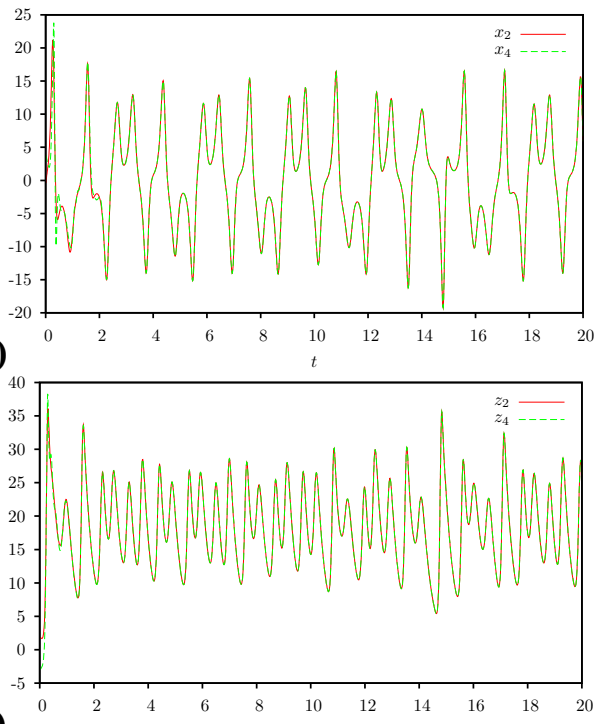

(c)

Figure 6: Evolution of the drive system (4.2) and response system (4.4) state, (a) signals $x_{2}$ and $x_{4} ;$ (b) signals $y_{2}$ and $y_{4}$; (c) signals $z_{2}$ and $z_{4} ;(\mathbf{d})$ signals $w_{2}$ and $w_{4}$.

(a)

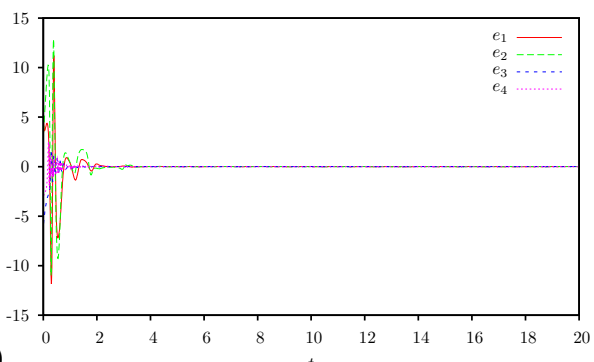

(b)

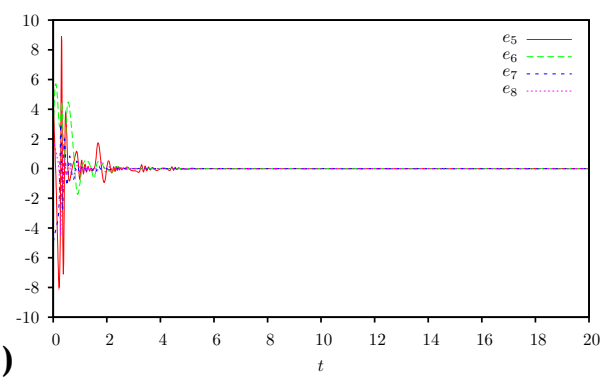

Figure 7: The dual synchronization errors (a) $e_{1}, e_{2}, e_{3}, e_{4}$ between the pair of systems (4.1) and (4.3); $(b) e_{5}, e_{6}, e_{7}, e_{8}$ between the pair of systems (4.2) and (4.4). (a)
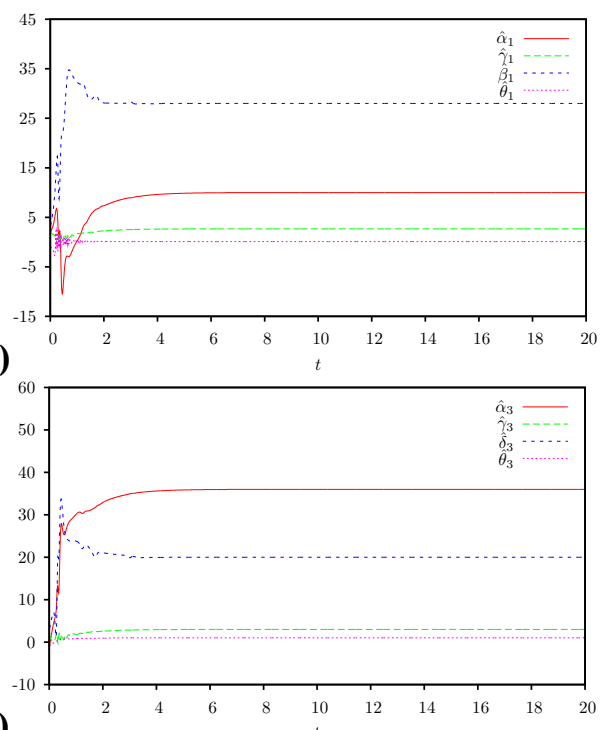

(b)
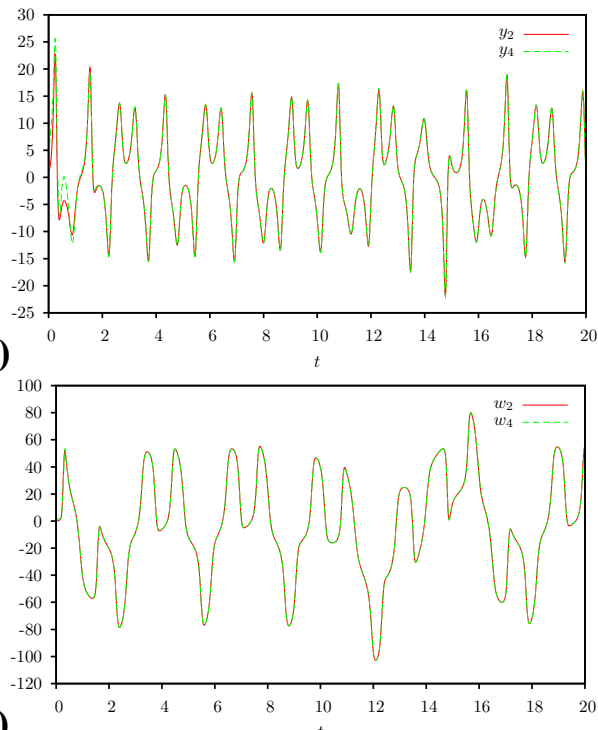

(d) 


\subsection{Numerical simulations}

In the numerical simulations, we demonstrate the effectiveness of the proposed method and discuss the simulation results for the adaptive dual synchronization of different chaotic systems, the fourth-order Runge-Kutta method is used to solve the systems (4.1)-(4.4) with time step size 0.001. The parameters of the drive and the response systems are set as $\alpha_{1}=10, \beta_{1}=28, \gamma_{1}=8 / 3, \theta_{1}=0.1, \alpha_{2}=35, \beta_{2}=$ $7, \delta_{2}=12, \gamma_{2}=3, \theta_{2}=0.6, \alpha_{3}=35, \delta_{3}=20, \gamma_{3}=3, \theta_{3}=1, \alpha_{4}=1, \beta_{4}=26, \gamma_{4}=0.7, \delta_{4}=1.5$ and $a_{i}=(1,1,1,1), b_{i}=(1,1,1,1), i=1,2,4$ and $k_{i}=4, i=0,1, \ldots, 8$ for which condition $P$ is positive definite. The initial conditions of the drive and response systems are taken as $x_{1}(0)=-1, y_{1}(0)=-1, z_{1}(0)=$ $1, w_{1}(0)=1, x_{2}(0)=-1, y_{2}(0)=1, z_{2}(0)=2, w_{2}(0)=0, x_{3}(0)=5, y_{3}(0)=2, z_{3}(0)=-5, w_{3}(0)=-2$ and $x_{4}(0)=-4, y_{4}(0)=3, z_{4}(0)=-3, w_{4}(0)=2$, respectively. Adaptive dual synchronization of the systems (4.1)-(4.4) via adaptive control law (4.6) and (4.7) with the initial estimated parameters $\hat{\alpha}_{1}(0)=$ $2, \hat{\beta}_{1}(0)-1=, \hat{\gamma}_{1}(0)=3, \hat{\theta}_{1}(0)=2, \hat{\alpha}_{2}(0)=3, \hat{\beta}_{2}(0)=2, \hat{\delta}_{2}(0)=-1, \hat{\gamma}_{2}(0)=3, \hat{\theta}_{2}(0)=2, \hat{\alpha}_{3}(0)=-1$, $\hat{\delta}_{3}(0)=3, \hat{\gamma}_{3}(0)=2, \hat{\theta}_{3}(0)=-1, \hat{\alpha}_{4}(0)=3, \hat{\beta}_{4}(0)=3, \hat{\gamma}_{4}(0)=3, \hat{\delta}_{4}(0)=3$ are shown in Figures 5-8. Figures 5-6 show the evolution of the drive systems (4.1)-(4.2) and the response systems (4.3)-(4.4). Figure 7 shows the error signals $e_{1}, e_{2}, e_{3}, e_{4}$ and $e_{5}, e_{6}, e_{7}, e_{8}$, between the pair of drive systems and the pair of response systems with initial conditions $e_{1}(0)=6, e_{2}(0)=3, e_{3}(0)=-6, e_{4}(0)=-3, e_{5}(0)=-3, e_{6}(0)=2, e_{7}(0)=$ $-5, e_{8}(0)=2$. Figure 8 shows that the estimates $\hat{\alpha}_{1}, \hat{\beta}_{1}, \hat{\gamma}_{1}, \hat{\theta}_{1}, \hat{\alpha}_{2}, \hat{\beta}_{2}, \hat{\delta}_{2}, \hat{\gamma}_{2}, \hat{\theta}_{2}, \hat{\alpha}_{3}, \hat{\delta}_{3}, \hat{\gamma}_{3}, \hat{\theta}_{3}, \hat{\alpha}_{4}, \hat{\beta}_{4}, \hat{\gamma}_{4}, \hat{\delta}_{4}$ of the unknown parameters converges to $\alpha_{1}=10, \beta_{1}=28, \gamma_{1}=8 / 3, \theta_{1}=0.1, \alpha_{2}=35, \beta_{2}=7, \delta_{2}=12, \gamma_{2}=$ $3, \theta_{2}=0.6, \alpha_{3}=, \delta_{3}=20, \gamma_{3}=3, \theta_{3}=1, \alpha_{4}=1, \beta_{4}=26, \gamma_{4}=0.7, \delta_{4}=1.5$ as $\mathrm{t} \rightarrow \infty$.

\section{Conclusions}

In this paper, based on the Lyapunov theorem of stability, an adaptive control strategy is proposed to achieve the dual synchronized among four non-identical unknown chaotic and four non-identical unknown hyperchaotic systems are investigated.The graphical results fully certified the theoretical findings. The error states converged to the origin with sufficient transient speed. The estimation of unknown parameters approached to the true values of the unknown systems parameters. Numerical experimental results further validated the robustness and effectiveness of the proposed methodology.

\section{References}

[1] C. K. Ahn, Anti-synchronization of Time-delayed Chaotic Neural Networks Based on Adaptive Control, Int. J. Theoretical Physics, 48 (2009), 3498-3509. 1

[2] M. M. Al-Sawalha, M. S. M. Noorani, Adaptive anti-synchronization of two identical and different hyperchaotic systems with uncertain parameters, Commun. Nonlinear Sci. Numer. Simul., 15 (2010), 1036-1047.

[3] M. M. Al-Sawalha, M. S. M. Noorani, Anti-synchronization of chaotic systems with uncertain parameters via adaptive control, Phys. Lett. A, 373 (2009), 2852-2857.

[4] M. M. Al-Sawalha, M. S. M. Noorani, Anti-synchronization of two hyperchaotic systems via nonlinear control, Commun. Nonlinear Sci. Numer. Simul., 14 (2009), 3402-3411.

[5] M. M. Al-Sawalha, M. S. M. Noorani, On anti-synchronization of chaotic systems via nonlinear control, Chaos Solitons Fractals, 42 (2009), 170-179.

[6] S. Bhalekar, V. Daftardar-Gejji, Synchronization of different fractional order chaotic systems using active control, Commun. Nonlinear. Sci. Numer. Simulat., 15 (2010), 3536-3546. 1

[7] G. Chen, X. Dong, From chaos to order, World Scientific Publishing Co., River Edge, (1998). 1

[8] A. Chen, J. Lu, J. Lü, S. Yu, Generating hyperchaotic Lü attractor via state feedback control, Physica A, 364 (2006), 103-110. 4

[9] G. R. Chen, T. Ueta, Yet another chaotic attractor, Internat. J. Bifur. Chaos Appl. Sci. Engrg., 9 (1999), 1465-1476. 3

[10] T. G. Gao, G. R. Chen, Z. Q. Chen, S. J. Cang, The generation and circuit implementation of a new hyper-chaos based upon Lorenz system, Phys. Lett. A, 361 (2007), 78-86. 4

[11] R. Genesio, A. Tesi, A harmonic balance methods for the analysis of chaotic dynamics in nonlinear systems, Automatica, 28 (1992), 531-548. 3

[12] D. Ghosh, Projective-dual synchronization in delay dynamical systems with time-varying coupling delay, Nonlinear Dynam., 66 (2011), 717-730. 1

[13] D. Ghosh, A. R. Chowdhury, Dual-anticipating, dual and dual-lag synchronization in modulated time-delayed systems, Phys. Lett. A, 374 (2010), 3425-3436. 1 
[14] S. Hassan, S. Mohammad, Dual synchronization of chaotic systems via time-varying gain proportional feedback, Chaos Solitons Fractals, 38 (2008), 1342-1348. 1

[15] W. Jawaadaa, M. S. M. Noorani, M. M. Al-sawalha, Robust active sliding mode anti-synchronization of hyperchaotic systems with uncertainties and external disturbances, Nonlinear Anal. Real World Appl., 13 (2012), 2403-2413. 1

[16] C. M. Jiang, F. F. Zhang, T. X. Li, Synchronization and antisynchronization of N-coupled fractional-order complex chaotic systems with ring connection, Math. Methods Appl. Sci., 41 (2018), 2625-2638.

[17] C. M. Jiang, F. F. Zhang, H. Y. Qin, T. X. Li, Anti-synchronization of fractional-order chaotic complex systems with unknown parameters via adaptive control, J. Nonlinear Sci. Appl., 10 (2017), 5608-5621.

[18] A. Khan, M. Shahzad, Synchronization of circular restricted three body problem with lorenz hyper chaotic system using a robust adaptive sliding mode controller, Complexity, 18 (2013), 58-64. 1

[19] J. La Salle, S. Lefschtg, Stability by Lyapunov's Direct Method with Application, Academic Press, New York, (1961). 3, 4

[20] N. Li, J. Cao, New synchronization criteria for memristor-based networks: Adaptive control and feedback control schemes, Neural Networks, 61 (2015), 1-9. 1

[21] Y. Li, W. K. S. Tang, G. Chen, Generating hyperchaos via state feedback control, Int. J. Bifurc. Chaos, 10 (2005), $3367-$ 3375. 4

[22] Y. Liu, P. Davis, Dual synchronization of chaos, Phys. Rev. E, 61 (2000), 2176-2179. 1

[23] E. N. Lorenz, Deterministic nonperiodic flow, J. Atmos Sci., 20 (1963), 130-141. 3

[24] J. H. Lü, G. R. Chen, S. C. Zhang, The compound structure of a new chaotic attractor, Chaos Solitons Fractals, 14 (2002), 669-672. 3

[25] A. C. J. Luo, A theory for synchronization of dynamical systems, Commun. Nonlinear Sci. Numer. Simul., 14 (2009), 1901-1951. 1

[26] D. Ning, J.-A. Lu, X. P. Han, Dual synchronization based on two different chaotic systems: Lorenz systems and Rossler systems, J. Comput. Appl. Math., 206 (2007), 1046-1050. 1

[27] K. S. Ojo, A. N. Njah, S. T. Ogunjo, Comparison of backstepping and modified active control in projective synchronization of chaos in an extended Bonhffervan der Pol oscillator, Pramana, 80 (2013), 825-835. 1

[28] A. O. Almatroud, M. S. M. Noorani, M. M. Al-Sawalha, Adaptive dual anti-synchronization of chaotic systems with fully uncertain parameters, Optik, 127 (2016), 10478-10489. 1

[29] A. O. Almatroud, M. S. M. Noorani, M. M. Al-Sawalha, Adaptive dual synchronization of chaotic and hyperchaotic systems with fully uncertain parameters, Optik, 127 (2016), 7852-7864.

[30] A. O. Almatroud, M. S. M. Noorani, M. M. Al-Sawalha, Dual anti-synchronization of hyperchaotic systems via nonlinear control, AIP Conference Proceedings, 1830 (2017), 020017.

[31] A. O. Almatroud, M. S. M. Noorani, M. M. Al-Sawalha, Dual synchronization of chaotic and hyperchaotic systems, J. Nonlinear Sci. Appl., 9 (2016), 4666-4677.

[32] A. O. Almatroud, M. S. M. Noorani, M. M. Al-Sawalha, Function projective dual synchronization of chaotic systems with uncertain parameters, Nonlinear Dyn. Syst. Theory, 17 (2017), 193-204.

[33] A. O. Almatroud, M. S. M. Noorani, M. M. Al-Sawalha, Function Projective Dual Synchronization with Uncertain Parameters of Hyperchaotic Systems, Int. J. System Dyn. Appl., 16 (2017), 1-16.

[34] A. O. Almatroud, M. S. M. Noorani, M. M. Al-Sawalha, Nonlinear feedback control for dual synchronization of chaotic systems, AIP Conference Proceedings, 1784 (2016), 050007. 1

[35] L. M. Perora, T. L. Carroll, Synchronization in chaotic systems, Phys. Rev. Lett., 64 (1990), 821-824. 1

[36] J. J. E. Slotine, W. Li, Applied Nonlinear Control, Prentice Hall, New Jersey, (1991). 2.1, 3, 4

[37] L. Stenflo, Generalized Lorenz equations for acoustic-gravity waves in the atmosphere, Phys. Scr., 53 (1996), 83-84. 4

[38] L. S. Tsimring, M. M. Sushchik, Multiplexing Chaotic Signals Using Synchronization, Phys. Lett. A, 213 (1996), 155166. 1

[39] A. Uchida, S. Kinugawa, T. Matsuura, S. Yoshimori, Dual synchronization of chaos in one-way coupled microchip lasers, Phys. Rev. E, 67 (2003), 8 pages. 1

[40] S. P. Wen, Z. G. Zeng, T. W. Huang, Adaptive synchronization of memristor-based Chua's circuits, Phys. Lett. A, 376 (2012), 2775-2780. 1

[41] J. Xiao, Z.-Z. Ma, Y.-H. Yang, Dual synchronization of fractional-order chaotic systems via a linear controller, The Scientific World Journal, 2013 (2013), 6 pages. 1

[42] C.-C. Yang, Adaptive synchronization of Lü hyperchaotic system with uncertain parameters based on single-input controller, Nonlinear Dynam., 63 (2011), 447-454. 1

[43] C.-C. Yang, Adaptive single input control for synchronization of a 4D LorenzStenflo chaotic system, Arab. J. Sci. Eng., 39 (2014), 2413-2426. 1

[44] L.-X. Yang, J. Jiang, Adaptive synchronization of drive-response fractional-order complex dynamical networks with uncertain parameters, Commun. Nonlinear Sci. Numer. Simul., 19 (2014), 1496-1506. 1

[45] L. Zhang, Uniform of four fractional-order nonlinear feedback synchronizations, Optik, 125 (2014), 4508-4512. 1 